\title{
Blass, R. 1990. Relevance Relations in Discourse. A Study with Special Reference to Sissala. Cambridge: Cambridge University Press.
}

La teoría pragmática de la relevancia, originalmente formulada por Dan Sperber y Deirdre Wilson en una serie de artículos (se puede encontrar una buena compilación en Davis, 1991) y en un influyente libro (Sperber \& Wilson 1986), se ha convertido en una poderosa teoría alternativa a tratamientos semióticos más tradicionales a los que estos autores se refieren como "modelos de código". Estos, retomando la tradición saussuriana de la relación entre significante y significado, son modelos para los que la finalidad de la pragmática parece reducirse a descubrir el código que el usuario linguiístico emplea para descubrir el significado (implícito o explícito) de un mensaje.

Sperber y Wilson plantean su teoría pragmática en la línea de los modelos inferenciales como son todos los que, de una u otra forma, se han ocupado, partiendo de las ideas de H. P. Grice, de la cuestión de los principios conversacionales ${ }^{1}$. Pero Sperber y Wilson, aunque valoran la contribución de Grice como un buen punto de partida para una teoría de la comunicación, entienden que es insuficiente. Por ejemplo, señalan, no da cuenta del papel de la selección contextual en el acto comunicativo. Este es quizá el punto más fuerte de la teoría de la relevancia que ellos proponen como un principio único, operativo en todo acto comunicativo y que se resume en la ya popularizada sentencia "todo acto de comunicación ostensional comunica la presunción de su propia relevancia óptima".

El criterio de consistencia con el principio de relevancia, para estos autores, garantiza que haya una sola interpretación para cada elocución. Es evidente que tal enfoque de una teoría pragmática, un enfoque en el que un solo principio es el centro de la comunicación y la cognición, supone un desafío intelectual importante frente a modelos más complejos. Un caso de aplicación de algunas de las nociones más importantes de la teoría de la relevancia para un análisis pragmático de una lengua es el libro objeto de esta reseña. 
El libro de Regina Blass se basa en su tesis doctoral Discourse Connectivity and Constraints on Relevance in Sissala (Universidad de Londres) y está dividido en ocho capítulos. Los dos primeros se dedican a cuestiones teóricas de índole general, tales como la definición de discurso, su relación con la pragmática, el contexto y la textualidad, así como a exponer diversos aspectos de la teoría de la relevancia, la cual, según la autora, se opone a las teorías discursivas sobre la coherencia. La exposición sigue -en estos capítulos y, de forma más dispersa, en el resto del libro- fielmente y de forma acrítica los postulados de Sperber y Wilson. Los seis capítulos restantes utilizan como excusa algunos aspectos de una lengua exótica del subgrupo gurunsí, el sissala, para elaborar cuestiones relativas a la teoría pragmática de Sperber y Wilson. Así, el capítulo tercero se dedica a estudiar la distinción de estos autores entre los usos interpretativo y descriptivo de las elocuciones y el modo cómo las partículas carentes de carga semántica contribuyen a crear condiciones de relevancia pragmática; los capítulos cuarto y quinto abordan la distinción entre partículas que aportan contenido proposicional (truth-conditional) y partículas que sólo actúan como guías del procesamiento (non-truth-conditional). La autora sugiere un nuevo enfoque de la tipología del uso de las partículas, que se puede basar en un estudio de los métodos por medio de los que una partícula colabora en la consecución de la relevancia contextual. En los capítulos sexto y séptimo se estudian algunos marcadores discursivos del sissala cuya función es la de ahorrar al oyente esfuerzo de procesamiento y ayudarle a encontrar el sentido del mensaje. También se muestra cómo el hablante puede explotar en el discurso la relación que existe entre la determinación genérica y la cuantificación universal. El capítulo octavo se dedica al estudio de la coordinación y de los efectos estilísticos en sissala, haciendo alguna referencia comparativa a los efectos pragmáticos de la coordinación en inglés (que surgen de fuentes no léxicas).

Al final del libro, la autora recapitula lo que ha sido, según ella, la doble intención de su estudio: por un lado, contribuir al análisis semántico y pragmático de una lengua exótica no estudiada hasta ahora; por otro, utilizar dicho análisis para mostrar la fuerza teórica del modelo pragmático de Sperber y Wilson. Podemos desechar, pues, la declaración inicial de intenciones expresada en la introducción, por la que se prometía mostrar (como objetivo principal) cómo la coherencia discursiva es un fenómeno pragmático más que semántico. De hecho, aunque ciertamente esta cuestión surge de vez en cuando, más que el objetivo a demostrar constituye el objeto aceptado de antemano.

Si nos atenemos al primer objetivo, es evidente que el lector podrá aprender algo sobre algunas partículas pragmáticas del sissala y contrastar su valor y uso con el de, pongamos, el inglés y el alemán, las otras lenguas de referencia que surgen esporádicamente en los comentarios del libro. Un pequeño inconveniente, sin embargo, es que no se nos dice el criterio de selección de dichas partículas. Así, cada capítulo da la impresión, un tanto lamentable, de discontinuidad respecto al resto. Por ejemplo, se dedica un capítulo entero a explicar que en sissala existe una partícula o marcador pragmático (ré) cuyo valor es interpretativo (no descriptivo), es decir, que sirve para 
atribuir (al modo del estilo indirecto) una creencia -aunque Blass explora otros usos relacionados-, valor que en esta lengua se ha gramaticalizado. La pretensión es, por un lado, mostrar que la diferenciación pragmática que Sperber y Wilson defienden en abstracto se ejemplifica en una lengua en el nivel gramatical y, por otro, que es superior a tratamientos anteriores de partículas similares de otras lenguas como meros "indicadores modales". Entonces, en el capítulo siguiente, se discute el valor de otro número de partículas pragmáticas en función de sus efectos contextuales. ¿Qué conexión existe con el anterior? No necesitamos abundar más sobre la cuestión. El lector puede sacar sus propias impresiones del somero repaso de contenidos que se ofrece más arriba.

Del segundo objetivo lo loable es su carácter de objetivo. Lo reprensible es su falta de consecución. Por una razón. Si bien el modelo de Sperber y Wilson es lo suficientemente poderoso como para dar cuenta de un gran número de fenómenos pragmáticos (¿universales?), no es el único modelo de análisis pragmático disponible. La autora, bien consciente de esto, tiene buen cuidado de señalar debilidades en el tratamiento de ciertos fenómenos por parte de otros modelos, pero el problema es que prácticamente para cada fenómeno escoge un modelo diferente ${ }^{2}$. Queda por ver cómo, por ejemplo, modelos discursivos como los elaborados por Walter Kintsch y Teun A. van Dijk, o teorías pragmáticas -como las de Kent Bach y Robert Harnish, o las de Geoffrey Leech, de corte Griceano- o incluso modelos lingüísticos funcionalistas -como el de Simon Dik-, darían cuenta del mismo rango de fenómenos (y de otros). Estos modelos y teorías también son capaces, en su ámbito de aplicación, de explicar la correlación existente entre la estructura discursiva y los efectos comunicativos, así como la relación entre estructura (y/o expresión) y su función. De hecho, como la propia Blass señala, dar cuenta de estas cuestiones es el desideratum de todo analista del discurso y no la exclusiva de ninguno. Así, pues, la gran carencia del estudio de Blass es el contraste teórico de los mismos aspectos en diversos modelos. Tal enfoque, por otra parte, habría dotado a un trabajo, por demás profundo y revelador en otros muchos aspectos, del elemento aglutinador que todo estudio serio requiere.

\section{REFERENCIAS}

Bach, K. \& Harnish, R. M. 1979. Linguistic communication and speech acts. Cambridge, Mass.: MIT Press.

Clark, H. H. \& Marshall, C. 1981. "Definite reference and mutual knowledge", en A. Joshi, B. Webber \& I. Sag (eds.). Elements of Discourse Understanding. Cambridge:

2 - En los dos primeros capítulos parece criticar, en general, los modelos de coherencia discursiva. Luego, se centra en cuestiones muy específicas de otros autores, como es el caso de la discusión sobre las partículas (epistémicas) con valor de "se dice", donde se opone la distinción interpretativol descriptivo de Sperber y Wilson a la de F. R. Palmer (1986) entre proposiciones que expresan juicios y proposiciones evidenciales (los dos sistemas de modalidad epistémica). Otras nociones que ataca, en favor de la teoria de la relevancia, son la noción de conocimiento mutuo utilizada por Clark y Marshall (1981) 
Cambridge University Press.

Davis, S. 1991. (ed.). Pragmatics. A Reader. Oxford, New York: Oxford University Press.

Leech, G. 1983. The Principles of Pragmatics. London: Longman.

Levinson, S. C. 1983. Pragmatics. Cambridge: Cambridge University Press.

Morton, A. 1986. "Domains of discourse and common-sense metaphysics", en C. Travis (ed.). Meaning and Interpretation. Oxford: Basil Blackwell.

Palmer, F. R. 1986. Mood and Modality. Cambridge: Cambridge University Press.

Sperber, D. \& Wilson, D. 1986. Relevance. Communication and Cognition. Oxford: Blackwell.

Francisco J. Ruiz de Mendoza Ibáñez

Colegio Universitario de La Rioja 